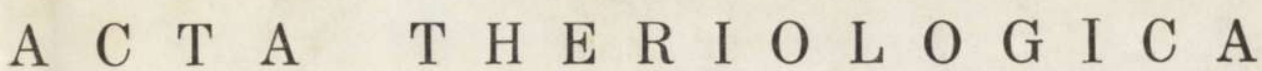

Egon SCHMIDT

\section{Ủber die mengenmässige Verteilung einiger Spitzmausarten in Ungarn}

\author{
[Mit 2 Tabellen und 2 Abb.]
}

\begin{abstract}
An ausgewählten Sammlungen von Schleiereulengewöllen aus Ungarn werden die mengenmässigen Verhältnisse des Auftretens von zwei Spitzmausgruppen untersucht: Sorex araneus and S. minutus (45 Sammelplätze) bzw. Crocidura suaveolens und C. leucodon (49 Sammelplätze). Der Autor diskutiret ökologische Anforderungen, die das mengenmässige Auftreten der untersuchten Arten in verschiedenen Gebieten Ungarns beeinflussen.
\end{abstract}

Vor kurzem habe ich die mengenmässige Verteilung der Genera Sorex Linnaeus, 1758 und Crocidura Wagler, 1832 durch Gewölluntersuchungen der Schleiereule Tyto alba (Scopoli, 1769) in Ungarn studiert ( $\mathrm{Schmidt}, 1971)$. Als Ergebnis habe ich bekommen, dass Sorex (vor allem S. araneus) in sehr hoher Dichte (über $80 \%$ ) nur im westlichen, alpin beeinflussten, feuchteren und kühleren Landesteil vorkommt. Westlich von Budapest, in NO-Transdanubien und in NordUngarn erreicht er eine mittlere Dichte $(40-60 \%$ gegenüber Crocidura). In der Tiefebene zwischen Donau und Theiss, daneben auch in SO-Transdanubien, war die Zahl der in den Gewöllen gefundenen Sorex Exemplaren schon bedeutend weniger (10-40\%), und in SO-Ungarn (der aridste Landesteil) kommt Sorex kaum mehr vor, in den Gewöllen wurden praktisch nur Crocidura gefunden.

Nach diesen globalen Ergebnissen scheint es mir als lohnend auch die mengenmässige Verhältnisse betreffend Sorex araneus Linnae us, 1758 und S. minutus Linnaeus, 1766, bez. Crocidura suaveolens ( $\mathrm{Pa} 1 \mathrm{las}$, 1811) und C. leucodon (H erm a n n, 1780) zu untersuchen. Inzwischen war das Material noch reicher geworden, d.h. es wurden Gewöllen von mehreren neuen Orten gesammelt. Für die Untersuchungen wurden nur Sammelplätze berücksichtigt, wo die Zahl der beiden Sorex 
Tabelle 1

Die mengenmässige Verteilung von Sorex araneus und $S$. minutus aus Schleiereulengewöllen, in Ungarn.

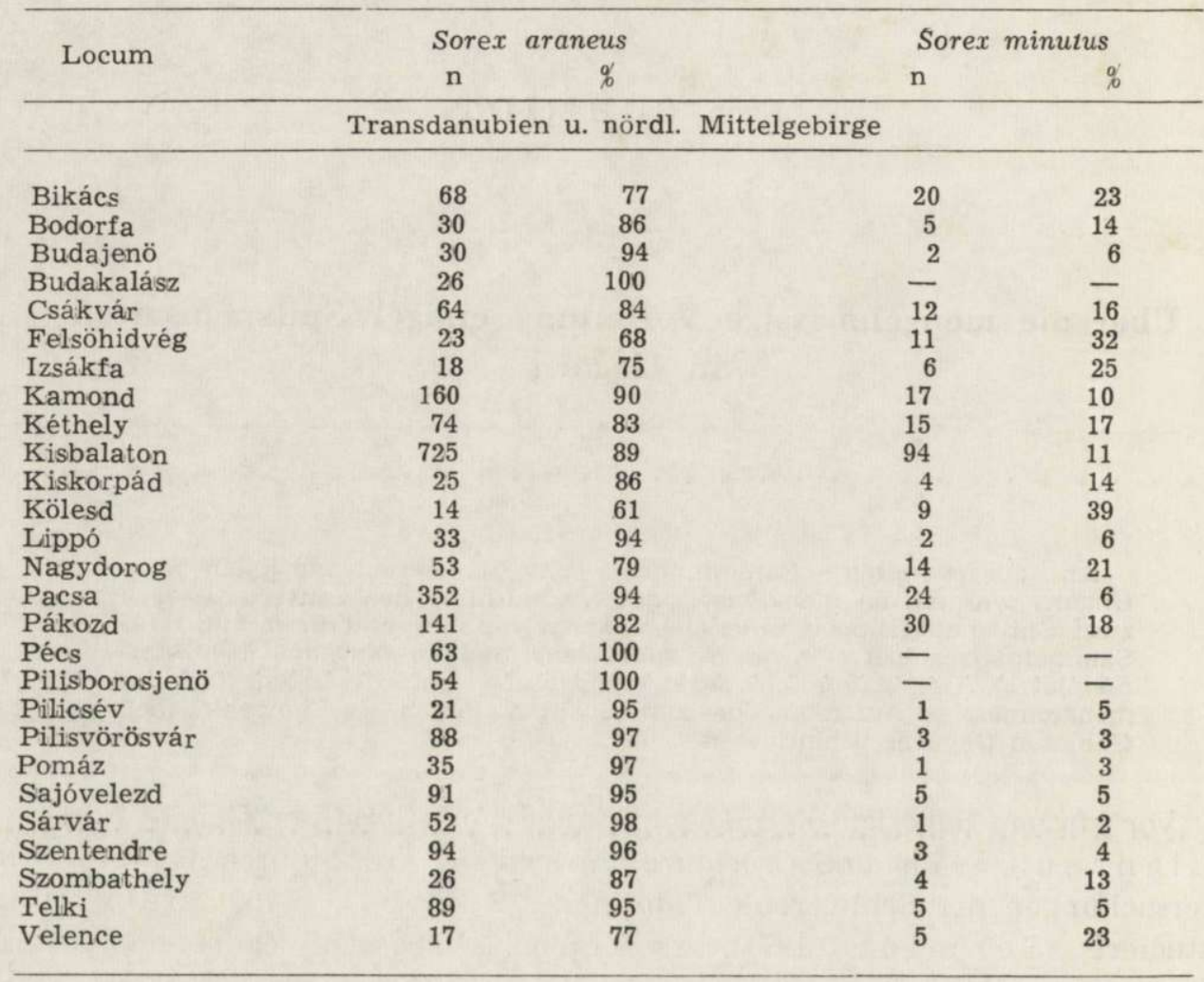

Tiefebene u. Hernád-Tal

\begin{tabular}{|c|c|c|c|c|}
\hline Baja & 48 & 96 & 2 & 4 \\
\hline Bácsalmás & 71 & 97 & 2 & 3 \\
\hline Dunakeszi & 135 & 100 & - & - \\
\hline Földes & 25 & 60 & 17 & 40 \\
\hline Hajdubagos & 17 & 68 & 8 & 32 \\
\hline Hernádszentandrás & 30 & 81 & 7 & 19 \\
\hline Jászladány & —* & - & 40 & 100 \\
\hline Katymár & 80 & 92 & 7 & 8 \\
\hline Méra & 17 & 85 & 3 & 15 \\
\hline Monostorpályi & 18 & 72 & 7 & 28 \\
\hline Nagyhalász & 74 & 96 & 3 & 4 \\
\hline Nagyiván & 139 & 64 & 78 & 36 \\
\hline Pánd & 68 & 85 & 12 & 15 \\
\hline Sövényháza & 42 & 51 & 41 & 49 \\
\hline Szeged & 10 & 48 & 11 & 52 \\
\hline Tetetlen & 13 & 54 & 11 & 46 \\
\hline Tornyosnémeti & 77 & 60 & 51 & 40 \\
\hline Vilmány & 20 & 56 & 16 & 44 \\
\hline
\end{tabular}

* unter 852 Kleinsäuger. 
Tabelle 2

Die megenmässige Verteilung von Crocidura leucodon und C. suaveolens aus Schleiereulengewöllen, in Ungarn.

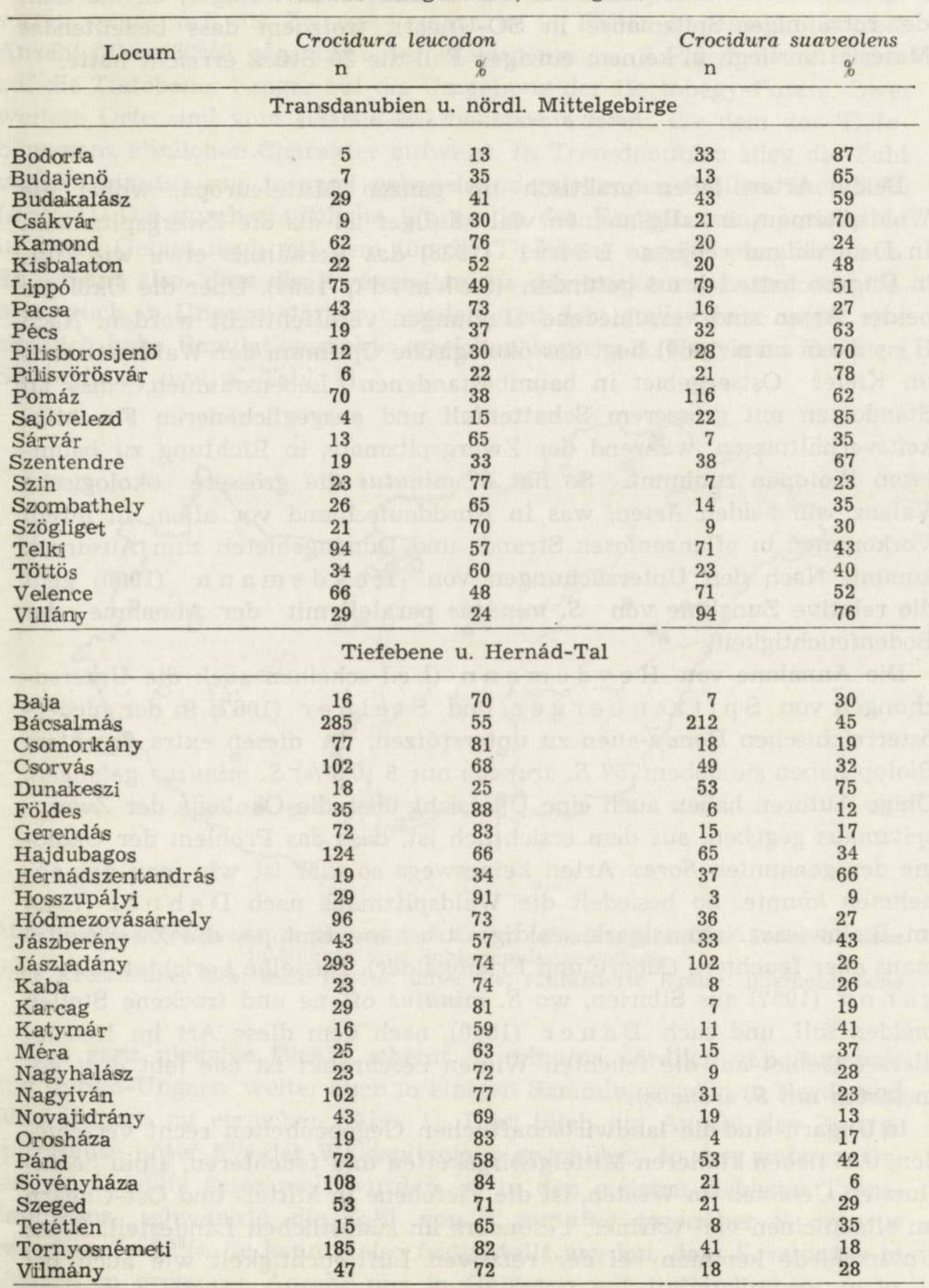


bez. Crocidura Arten mindestens 20-20 Stück war. Von der ersten Gruppe stehen mir 45, von der zweiten 49 Sammlungsorte zur Verfügung (Tab. 1 und 2). Die Sammelplätze von Sorex sind etwas weniger, da die Zahl der rotzähnigen Spitzmäuse in SO-Ungarn trotzdem dass bedeutendes Material vorliegt, in keinem einzigen Fall die 20 Stück erreicht hatte.

$$
\text { Sorex araneus - Sorex minutus }
$$

Beide Arten leben praktisch im ganzen Mitteleuropa, wobei die Waldspitzmaus im allgemeinen viel häufiger ist als die Zwergspitzmaus. In Deutschland schäzte Löhrl (1938) das Verhältnis etwa wie 10:1, in Ungarn hatte ich 6:1 gefunden ( $\mathrm{Schmidt}$, 1969). Über die Ökologie beider Arten sind verschiedene Meinungen veröffentlicht worden. Nach H e y d e m a n n 1960) liegt das ökologische Optimum der Waldspitzmaus im Kieler Ostseegebiet in baumbestandenen Lebensräumen, also an Standorten mit grösserem Schattenfall und ausgeglicheneren Feuchtigkeitsverhältnissen, während der Zwergspitzmaus in Richtung zu baumlosen Biotopen zunimmt. So hat $S$. minutus die grössere ökologische Valenz von beiden Arten, was in Norddeutschland vor allem in ihrem Vorkommen in pflanzenlosen Strand- und Dünengebieten zum Ausdruck kommt. Nach den Untersuchungen von Heydemann (1960) geht die relative Zunahme von S. minutus paralell mit der Abnahme der Bodenfeuchtigkeit.

Die Annahme von $\mathrm{Heydem}$ ann (l.c.) scheinen auch die Untersuchungen von Spitzen berger und Steiner (1967) in der niederösterreichischen Donau-auen zu unterstützen. In diesen extra feuchtem Biotop haben sie neben 757 S. araneus nur $6(0,8 \%)$ S. minutus gefangen. Obige Autoren haben auch eine Übersicht über die Ökologie der Zwergspitzmaus gegeben, aus dem ersichtlich ist, dass das Problem der Ökologie der genannten Sorex Arten keineswegs so klar ist wie man das annehmen könnte. So besiedelt die Waldspitzmaus nach D e h n el (1949) im Bialowieża Nationalpark waldige, trockene Biotope, die Zwergspitzmaus aber feuchtere (Moore und Erlenwälder). Dasselbe berichtet S $\mathrm{tr}$ og a n ov (1957) aus Sibirien, wo $S$. minutus offene und trockene Stellen meiden soll, und such B a u e $\mathrm{r}$ (1960), nach dem diese Art im Neusiedlersee-Gebiet auf die feuchten Wiesen beschränkt ist (sie lebt dort gemeinsam mit $S$. araneus).

In Ungarn sind die landwirtschaftlichen Gegebenheiten recht verschieden, d.h. neben kühleren Mittelgebirgsketten und feuchteren, alpin beeinflussten Gebieten im Westen, ist die Tiefebene in Mittel- und Ost-Ungarn im allgemeinen viel wärmer, kesonders im südöstlichen Landesteil. Diese Un'erschiede kommen bei der relativen Luftfeuchtigkeit wie auch bei 
durchschnittlichen Wärme deutlich im Ausdruck. Von dem 45 Sammlungsorten zeigte sich die Zwergspitzmaus in 11 Fällen in ziemlich grosser Anzahl (über $30 \%$ ) gegenüber der Waldspitzmaus. 7 Plätze davon fielen auf die Tiefebene, 4 sogar auf die Umgebung der Hortobágy-Puszta. Zwei weitere Orte sind vom Hernád-Tal bekannt worden, die dem der Tiefebene ganz ähnlichen Charakter aufweist. In Transdanubien stieg die Zahl von $S$. minutus nur in zwei nebeneinanderstehenden Stellen über $30 \%$ (Abb. 1). Die durchschnittliche Wärme in der Fortpflanzungsperiode ist in disem Gebiet noch mit dem für die Tiefebene angegebenen identisch. Es scheint also, dass die Zwergspitzmaus die trockenen, baumlosen Gebiete auch in Ungarn stärker besiedelt und dort stellenweise sogar eine ziemlich hohe Populationsdichte erreichen kann (z.B. Nagyiván, Tetétlen, Sövényháza, usw. s. Tabl. 1).

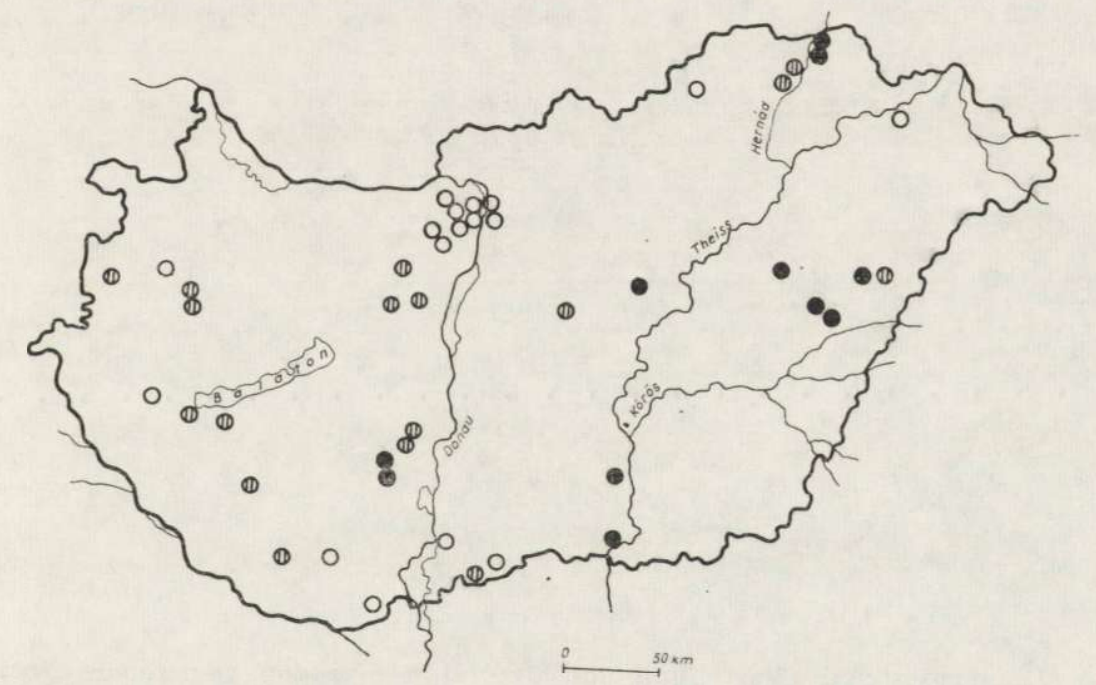

Abb. 1: Die prezentuelle Verteilung von Sorex minutus gegenüber Sorex araneus in Ungarn (aus Schleiereulengewöllen).

Volle Kreise: über $30 \%$; leere Kreise: unter $6 \%$; schraffierte Kreise: überbebliebene Werte.

Eine ganz niedrige Dichte scheint $S$. minutus nördlich von Budapest und in Süd-Ungarn, weiter auch in einigen Sammlungsorten in Nord- und West-Ungarn zu erreichen (Abb. 1). Hier blieb die Anzahl der Zwergspitzmäuse unter $6 \%$ der Waldspitzmaus gegenüber. In den anderen Orten wo Gewölle gesammelt wurden, so in den meisten Gebieten Transdanubiens, schwankte die $\mathrm{Zahl}$ von $S$. minutus gegenüber $S$. araneus zwischen $7-29 \%$. Es konnte also festgestellt werden, dass $S$. minutus in Ungarn in grösserer Anzahl nur in Gebieten mit tiefländischen, mehr baunmlosen und trockenen Charakter vorkommt. Durchschnittlich war Acta theriol. 19 
sie in den Gewöllen in Transdanubien und im nördlichen Hügelland in $19,3 \%$ gegenüber $S$. araneus vertreten, dasselbe Verhältnis war in der Tiefebene $31,6 \%$.

$$
\text { Crocidura suaveolens - Crocidura leucodon }
$$

Über die Ökologie beider Arten sind in der Literatur ebenfalls abweichende Angaben zu finden. Nach R i ch te r (1963) kann man die ökologischen Ansprüche nur schwer zu unterscheiden, obzwar leucodon von allen mitteleuropäischen Crocidura Arten diejenige ist, die die grösste Trockenheit verträgt und der auch die Monokultursteppe bewohnt. Dage-

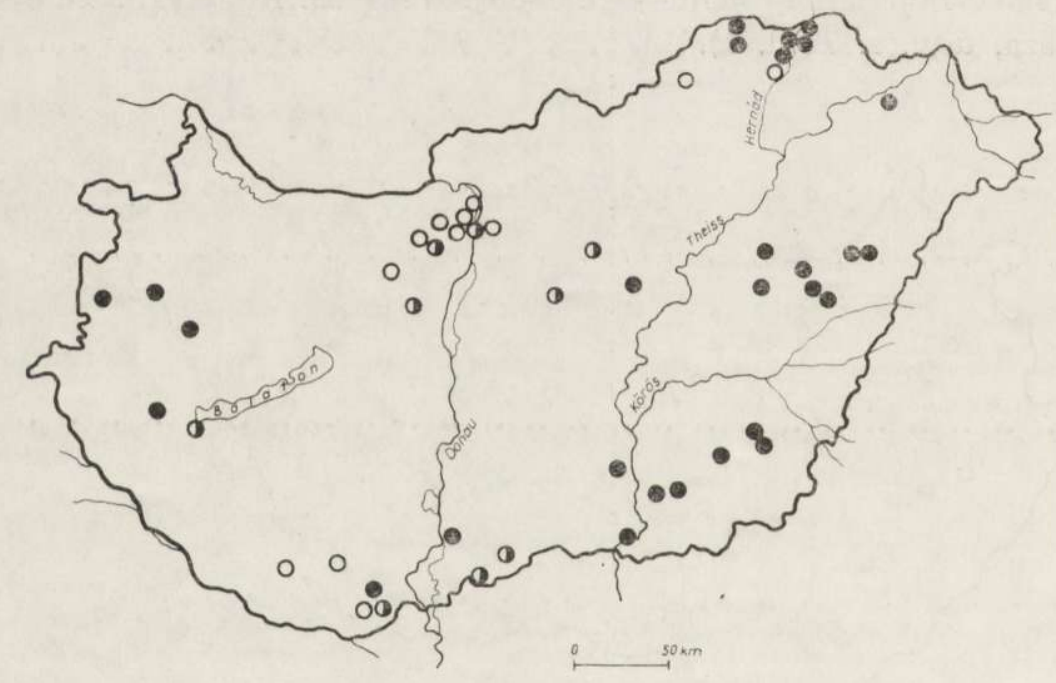

Abb. 2: Die prozentuelle Verteilung von Crocidura leucodon gegenüber Crocidura Volle Kreise: über 60\%; leere Kreise: unter $40 \%$; schraffierte Kreise: übergebliebene Werte.

gen scheint $C$. suaveolens etwas feuchtere und schattigere Plätze zu bevorzugen. Nach Richter (1963) lebt sie vorwiegend in bebautem Gelände und wird fernab von Siedlungen nur selten angetroffen. Dagegen konnte B a u e $\mathrm{r}(1960)$ suaveolens im Neusiedlersee-Gebiet auch in ganz siedlungsfernen Biotopen gefunden, und betont, dass die beiden Arten in ihrem Lebensraum nicht kategorisch voneinander getrennt werden können. Auch meine eigene Erfahrungen sind diesbezüglich verschieden. In Apaj-Puszta, in einem echten Puszta-Gebiet südöstlich von Budapest z.B. habe ich in Steinkauzgewöllen nur suaveolens gefunden $(\mathrm{S} \mathrm{ch} \mathrm{midt}$ : 1962). Hier soll die Gartenspitzmaus die menschenlose Puszta (Festuce 
tum pseudovina-Assoziation) zu bewohnen. Dagegen zeigten die Gewöllen aus der Umgebung der Hortobágy-Puszta und aus SO-Ungarn eine Überzahl von leucodon. Betreffend der mengenmässigen Verteilung beider Arten in Ungarn (S c h m i d t, 1967) konnte das früheres Bild im Spiegel mehrerer neuer Sammlungen (vor allem in der Tiefebene) ergänzt werden. Laut diesem ist die Feldspitzmaus östlich der Theiss, aber auch in den Hernád- und Bodrog-Tälern in Uberzahl (Abb. 2). Dieselben Verhältnisse zeigten die Gewöllangaben auch in dem wesentlich kühleren und feuchteren West-Ungarn, eine ökologische Absonderung konnte also nicht festgestellt werden.

Die Gartenspitzmaus scheint in Ungarn nur in zwei Gebieten die Feldspitzmaus überzutreffen, nämlich in der Umgebung von Budapest und in SO-Transdanubien. Praktisch dieselben Gebiete zeigten die Minimalwerte von S. minutus (Abb. 1), so scheint die Ökologie beider Arten gegensätzlich zu sein.

Gruppiert man die erhaltenen Resultate so kommt C. leucodon mit grösstem Populationsdichte östlich der Theiss, in der Hortobágy-Gegend und in SO-Ungarn vor. Fast die gleichen Werte zeigten die Angaben aus dem Hernád-Tal, was in ihrem Charakter dem vorigen entspricht. Von der Tiefebene zwischen Donau und Theiss hatte ich nur von den nördlichen und südlichen Teilen Material bekommen (die Schleiereule scheint in den mittleren Gebieten sehr selten zu sein), das Verhältnis zwischen C. leucodon und C. suavelens war in den untersuchten Stellen praktisch das gleiche. Wie das die Gewöllfunde eindeutig zeigten ist die Gartenspitzmaus in der Umgebung von Budapest in der Mehrzahl $(60 \% \mathrm{im}$ Durchschnitt gegenüber C. leucodon). Praktisch dieselbe Situation war auch in SO-Transdanubien zu finden $(61,8 \%$ gegenüber $C$. leucodon $)$. In West-Ungarn zeigte sich endlich die Feldspitzmaus mit $67,8 \%$ wieder als dominante Art von der beiden. Nur im Kisbalaton-Gebiet war die mengenmässige Verteilung beider Arten die gleiche (Tab. 2).

\section{SCHRIFTTUM}

1. B a u e r K., 1960: Die Säugetiere des Neusiedlersee-Gebietes. Bonn. zool. Beitr. 11: $141-344$.

2. De hnel A., 1949: Badania nad rodzajem Sorex. L. Annls Univ. Mariae CurieSkłodowska, Sect. C 4, 2: 16-102.

3. He y demann B., 1960: Zur Ókologie von Sorex araneus L. und Sorex minutus L. Z. f. Säugetierk. 25: 24-29.

4. Lö hrl H., 1938: Studien an einheimischen Muriden und Soriciden. Z. f. Säugetierk. 13: $114-160$.

5. Richter H., 1963: Zur Verbreitung der Wimperspitzmäuse (Crocidura W a gl e r, 1832) in Mitteleuropa. Zool. Abh. Mus. Tierk., Dresden, 26: 219-242. 
6. Schmidt E., 1962: Angaben über die Kleinsäugetierfauna in der Umgebung von Apaj-Puszta. Vertebr. Hung., 4: 83-91.

7. Schmidt E., 1967: Vergleichende und populationsstatistische Untersuchungen an Unterkiefern der Feld- und Gartenspitzmaus, Crocidura leucodon (Hermann, 1780) und Crocidura suaveolens ( $\mathrm{Palla} \mathrm{s}, 1811)$, in Ungarn. Säugetierk. Mitt. 15: $61-67$.

8. S chmidt E., 1969: Daten zur Verbreitung einiger Kleinsäugerarten in Ungarn, an Hand von Eulengewölluntersuchungen (Vorläufiger Bericht). Vertebr. Hung. 11: $137-153$.

9. S chmidt E., 1971: Beispiele zur Bedeutung von Gewöllenuntersuchungen für die Kenntnis der Kleinsäugerwelt in einem engeren tiergeographischen Bezirk (Ungarn). Säugetierk. Mitt., 19: 44-48.

10. Spitzenberger F. \& Steiner H., 1967: Die Okologie der Insectivora und Rodentia (Mammalia) der Stockerauer Donau-Auen (Niederösterreich). Bonn. zool. Beitr. 18: 258-296.

11. Strog a n ov S. U., 1957: Zveri Sibiri. Nasekomojadnyje. Izd. Ak. Nauk SSSR: 1-267, Moskva.

Accepted, March 30, 1973.

Madártani Intézet,

Budapest XII, Költó utca 21,

Ungarn.

Egon SCHMIDT

\section{STOSUNKI ILOSCIOWE ROZMIESZCZENIA RYJÓWKOWATYCH NA WĘGRZECH}

Streszczenie

W wybranych zbiorach wypluwek płomykówki z obszaru Węgier zbadano wzajemne stosunki ilościowe występowania 2 par gatunków ryjówkowatych: Sorex araneus i S. minutus (45 zbiorów) oraz Crocidura suaveolens i C. leucodon (49 zbiorów). Autor dyskutuje wymogi ekologiczne, wpływające na częstość występowania badanych gatunków. 